\title{
Beam Properties of the new Radiation Effects Research Stations at Indiana University Cyclotron Facility
}

\author{
B. von Przewoski, T. Rinckel, W. Manwaring, G. \\ Broxton, M. Chipara,T. Ellis, E.R. Hall, A. Kinser \\ Indiana University Cyclotron Facility \\ Bloomington, IN 47405, USA
}

\author{
K.M. Murray \\ KM Sciences \\ Basking Ridge, NJ 07920, USA
}

\begin{abstract}
We describe two new beamlines for radiation effects research at the Indiana University Cyclotron Facility. Protons with energies up to $205 \mathrm{MeV}$ are available. One of the beamlines offers momentum selected beams at energies as low as $52 \mathrm{MeV}$. Beam characteristics such as transmission, energy, energy spread and lateral profile are described and compared to calculations. The dosimetry with emphasis on the lower energies is discussed.
\end{abstract}

\section{INTRODUCTION}

High energy protons are useful for ground based simulation of the effects of space radiation on electronic components, devices and systems as well as passive materials. The proton beam also allows the simulation of radiation environments as they exist near colliding beams used in particle physics research. Finally, the proton beam is a useful tool for detector development and calibration.

The rationale for proton testing for space applications is twofold. Firstly, protons are the most prolific ion species in space. Secondly, it has been shown that characterization of the single event response of a device or system to accelerator produced protons with energies above $100 \mathrm{MeV}$ can be used to predict the single event response to heavy ion exposures of devices [1] and determine a firm upper bound to the on-orbit heavy ion upset rate and failure risk [2]. Given the high cost of high energy heavy ion testing, the convenience of testing in air without the need to remove device lids, the relatively small angular spread due to multiple scattering in passing through several layers of components, and the fact that, for protons, good rate prediction depends principally on accurate high energy asymptotic cross section data [1] lead to an increased demand for testing with high energy protons.

The Indiana University Cyclotron Facility (IUCF) is located in Bloomington, Indiana, and provides proton beams of up to $205 \mathrm{MeV}$ for a variety of applications. It was originally built and operated as a laboratory for medium energy nuclear physics. Today, IUCF houses a clinical

\author{
C.C. Foster \\ Foster Consulting Services, LLC \\ Tacoma, WA 98466, USA
}

facility for proton therapy and two dedicated beamlines for proton radiation testing [3]. IUCF has had an active protonbased radiation effects research program since 1994.

Two new, state-of-the art radiation effects research stations (RERS1 and RERS2) and a dedicated counting room have been installed and fully instrumented. The first beamline became operational in July 2003 while the second beamline was ready for beam delivery in December of 2003. Fig. 1 shows the layout of IUCF including projects under construction. The two new beamlines (RERS1/2) were sponsored by the NASA Johnson Space Center. Also shown are the Midwestern Proton Therapy Institute (MPRI) and the future neutron facility consisting of LENS (Low Energy Neutron Source) and NREP (Neutron Radiation Effects Program) exposure area. The capabilities of the LENS and NREP will be described more fully later in this paper.

The Radiation Effects Research Program offers flexible scheduling, user-friendly facilities and state-of-the art dosimetry. Access to the proton beam is commercially available to industry, government, military and academic users 365 days a year. The present paper will review those facilities, report on beam properties and validation studies for the proton dosimetry and briefly describe the future neutron radiation effects research facility.

\section{RERS OVERVIEW}

Beam is extracted from the cyclotron at $205 \mathrm{MeV}$ and delivered by a fast kicker system to each of the new irradiation stations. Each kicker system consists of a fast ferrite kicker magnet and a Lambertson magnet [4]. A Lambertson magnet is a magnet designed to have a high field region separated by a small distance ( a millimeter or so) from a very low field region. The kicker magnet deflects the proton beam from the low field region to the high field region to direct it from one beam line to another. The beamlines are designed to eventually allow rapid beam sharing with the medical facility (MPRI) and between RERS1/2.

Available fluxes are between $10^{2}$ and $10^{11}$ protons $/$ second $/ \mathrm{cm}^{2}$ (at $205 \mathrm{MeV}$ ). RERS2 offers momentum selected beams at energies as low as $52 \mathrm{MeV}$. For each irradiation, the fluence is automatically determined 


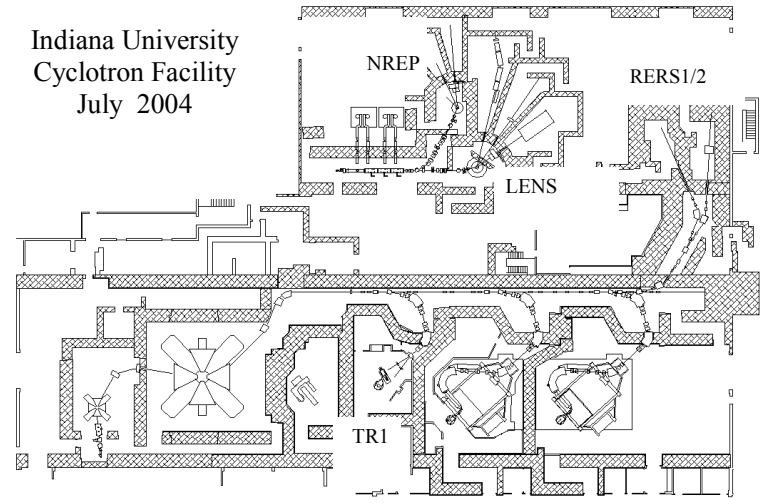

Figure 1. Facility Layout with the two Radiation Effects Research Stations (RERS1/2), the LENS/NREP project which is under construction and the medical facility (MPRI) where TR1 denotes the first operational treatment room. The so-called trunkline runs horizontally from left to right.

from the radiation time, the average proton current measured with a calibrated secondary electron monitor, and the known beam profile. All of this is logged as is the incident exposure dose to the device calculated from the fluence and stopping power for protons in the material of the device at the incident proton energy. Both beamlines feature exposure times as short as a few seconds and as long as 12 hours, and beam spot sizes from $2 \mathrm{~cm}$ in diameter to $7 \mathrm{~cm}$ in diameter. Beam spot sizes as large as $30 \mathrm{~cm}$ diameter are available in RERS1. Tab. 1 summarizes the proton capabilities of the IUCF Radiation Effects Research Stations.

\section{RADIATION EFFECTS RESEARCH STATIONS}

The radiation effects research stations (RERS1/2) are shown schematically in Fig. 2. The setup has been described in detail elsewhere [5] and will only be briefly reviewed here.

An upstream beam stop (1) is used to adjust the desired beam current prior to an irradiation and admits the beam to the RERS when removed from the path of the beam.

A copper (tantalum) foil which is inserted into the beam at position 2 (3) spreads the beam by multiple scattering. The collimator at position (4) defines the beam at the position of the DUT. The copper spreader at position 2 provides a $7 \mathrm{~cm}$ diameter beam spot as described in reference [5]. A tantalum spreader at position (3), which is much closer to the collimator at position (4), is used to produce a large area, up to $30 \mathrm{~cm}$ diameter, beam spot. Immediately following the defining collimator is the secondary emission monitor (SEM), consisting of 15 half mil thick $\mathrm{Cu}$ foils alternately biased to collect the secondary electrons produced by the proton beam as it passes through. A copper beam stop or Faraday Cup (5) is located downstream from the SEM. The SEM measures the beam current and thus the fluence for each exposure. It is calibrated prior to each exposure by measuring the ratio of current from the Faraday Cup (5) to the current from the SEM. Both currents are measured with
TABLE I. CAPABILITIES OF THE RADIATION EFFECTS RESEARCH PROGRAM (RERP)

$\begin{array}{ll}\text { Energy Range: } & 30 \text { to } 200 \mathrm{MeV} \\ \text { Flux: } & 10^{2} \text { to }>10^{11} \mathrm{p} / \mathrm{s} / \mathrm{cm}^{2} \\ \text { Areas: } & <2 \mathrm{~cm} \text { to } 30 \mathrm{~cm} \text { diameter } \\ \text { Uniformity: } & <40 \% \text { variation over area } \\ \text { Absolute Dosimetry: } & \text { Better than } 10 \% \text { routinely } \\ \text { Exposure Durations: } & 5 \mathrm{~s}-\text { several h } \\ \text { Convenience: } & \begin{array}{l}2 \text { minutes per room entry, remote } \\ \text { device positioning, flexible } \\ \end{array} \\ & \text { scheduling }\end{array}$

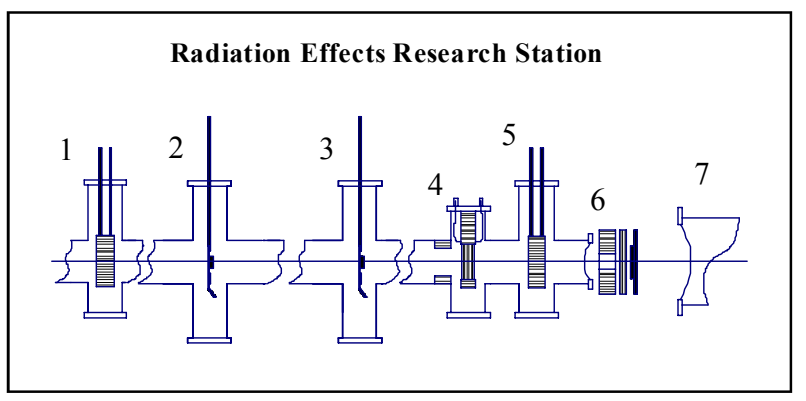

Figure 2. Schematic view of the RERS. The proton beam goes from left to right. Shown are the movable upstream beam stop (1), beam spreader target ladders $(2,3)$, dosimetry secondary electron monitor (4), movable dosimetry beam stop (5), air gap with external collimator, energy degrader and device under test (6), and beam dump (7). The drawing is not to scale. The distance from (2) to (5) is about $200 \mathrm{~cm}$ and the distance from (5) to (6) may be as much as $350 \mathrm{~cm}$.

computer controlled picoammeters (Keithley 6485). An external $5.08 \mathrm{~cm}$ thick copper collimator or a copper degrader may be inserted in air between the exit window and the DUT.

A new dosimetry software package was developed on an alpha (VMS) platform consistent with standard IUCF control software.

Beam stops (1) and (5) are remotely controlled in the proper sequence to calibrate the SEM before each exposure and to start and stop the irradiation of the DUT. The duration of the irradiation may be pre-selected in terms of fluence, dose or time. For each irradiation the dose and fluence are automatically determined and logged. Parameters for each irradiation such as beam energy, beam current, picoammeter range, stopping power in silicon and SEM calibration factor are also recorded.

\section{ENERGY SELECTION}

Both beamlines have the option to degrade the beam energy immediately upstream of the DUT as reported earlier [5]. In RERS1 such degraders are mounted on a remotely operated degrader positioner which has room for up to four degraders. If this positioner is used it only takes a minute or less to change the energy. Although this is convenient, it 
introduces uncertainties from the increased energy spread due to straggling and geometric spreading from multiple scattering of the protons. The geometric spreading of the beam downstream of the degrader is corrected for in the dosimetry software.

RERS2 has Beryllium degraders well upstream of the radiation effects station which may be used to degrade the beam energy to $52,72,102$, or $149 \mathrm{MeV}$. A $16.5 \mathrm{~cm}$ thick rod of beryllium is needed to degrade the beam to $52 \mathrm{MeV}$. Beryllium was chosen for its small multiple scattering angle [6], thus limiting the loss of beam outside the momentum acceptance of the beamline following the degrader. Momentum analysis by a dipole and slit system following the degrader guarantees nearly mono-energetic beams at the location of the DUT. The beryllium degrader and the slits are remotely controlled. Energy dependent magnet currents are loaded from data files stored in the controls computer. Thus, an energy change of the beam is accomplished in about 30 minutes. Neither the beryllium degrader nor the dipole and slit system are located in the irradiation room. By placing an additional degrader immediately upstream of the DUT in RERS2, a beam energy as low as desired can be obtained at the cost of increased energy spreading.

Whenever the beam energy is degraded, the maximum available flux is reduced. Due to the geometric spreading of the beam after the degrader the number of protons $/ \mathrm{s} / \mathrm{cm}^{2}$ is reduced. In RERS1 the flux reduction due to the presence of the degrader is negligible while in RERS2 the maximum available flux is about two orders of magnitude lower at 52 $\mathrm{MeV}$ than at $205 \mathrm{MeV}$.

\section{BEAM ENERGY CALIBRATION}

The beam energy can be determined using a calibrated multi-leaf Faraday Cup (MLFC) which is placed at the location of the DUT. The MLFC consists of 30 layers of 0.5 $\mathrm{mm}$ thick Al leaves alternating with $80 \mu \mathrm{m}$ thick kapton sheets sandwiched between a front and a back cover plate. The protons stop in the Al layers and the current from each $\mathrm{Al}$ sheet is read out. The highest current is measured where the beam ranges out. The beam energy can be determined from the number of $\mathrm{Al}$ layers that the protons are able to penetrate. The MLFC is built such that a $50 \mathrm{MeV}$ proton stops approximately in the central layer. For higher proton energies additional copper degraders are placed in front of the MLFC.

Fig. 3 illustrates the difference in beam energy spread for the lowest available energy $(\sim 50 \mathrm{MeV})$ in both beamlines. The larger width of the curve labeled RERS1 is due to the presence of the degrader immediately upstream of the DUT. The width of the curve labeled RERS2 does not represent the beam energy spread, which is much smaller. Instead, the width is due to straggling in the front plate and the $\mathrm{Al}$ of the MLFC itself.

The energy of the beam at the DUT can be determined from energy loss calculations in two ways. 1.) An energy loss calculation is performed where the energy at the DUT is treated as a parameter which is optimized such that the beam stops in the $\mathrm{Al}$ layer as determined from the MLFC

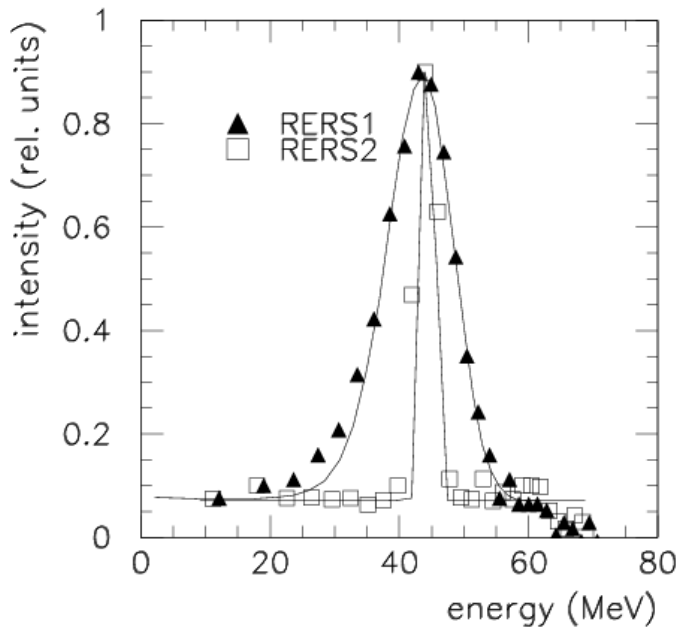

Figure 3. Multi Leaf Faraday Cup spectra (see section about degraded beams) for degraded beams in RERS1 and RERS2. The difference in width between the two curves is due to the presence of the degrader immediately upstream of the DUT in RERS1. The lines are SRIM calculations

spectrum. 2.) The energy of the beam extracted from the cyclotron as determined by an identical MLFC in the trunkline (see Fig.1) is used and the energy loss in the Be degrader is taken into account to calculate the energy at the DUT. Tab. 2 lists the energies obtained both ways. The two approaches give consistent results. The error estimate is based on the uncertainty introduced by different iteration methods in the energy loss calculations. The error is smallest when no energy loss calculation in a thick layer of material is required to determine the energy at the DUT, since uncertainties in the calculations increase with material thickness. All calculations were based on material densities from the SRIM code [7], which was one of the software packages used to calculate the energy loss.

\section{TRANSMISSION}

The transmission through a beamline is defined as that fraction of incident protons that arrive at the end of the beamline. The measured transmission for RERS1 and RERS2 at $205 \mathrm{MeV}$ is better than $99 \%$.

The flux in RERS2 decreases at lower energies, because the momentum selection system only transmits that portion of the beam which has the desired energy downstream of the beryllium degrader. Fig. 4 shows the measured ratio of the beam current downstream of the momentum selection system of RERS2 (Fig. 2, (5)) vs current on the beam stop immediately downstream of the degrader (Fig. 2, (1)). No spreader was used for this measurement. For comparison, the transmission through the first of IUCF's medical beamlines (Fig.1, TR1), which has a similar degrader system, is also shown (solid squares). The line is a calculation for a constant momentum acceptance $\Delta \mathrm{p} / \mathrm{p}$ of the beamline. It describes the observed energy dependence fairly well. 
TABLE II ENERGIES WITHOUT DEGRADER AND WITH THE FOUR BE DEGRADERS. THE SLIGHTLY DIFFERENT ENERGIES PER DEGRADER CORRESPOND TO DIFFERENT SPREADERS. THE LEFT COLUMN CORRESPONDS TO THE ENERGY AT THE DUT AS CALCULATED FROM THE MLFC AT THE END OF THE RERS2 BEAMLINE. THE RIGHT COLUMN WAS OBTAINED FROM ENERGY LOSS CALCULATIONS USING THE STARTING ENERGY AS MEASURED BY THE TRUNKLINE MLFC.

\begin{tabular}{|c|c|}
\hline $\begin{array}{l}\mathrm{T}(\mathrm{MeV}) \\
\text { from } \\
\text { RERS2 } \\
\text { MLFC }\end{array}$ & $\begin{array}{c}\mathrm{T}(\mathrm{MeV}) \\
\text { from } \\
\text { energy loss } \\
\text { in } \mathrm{Be}\end{array}$ \\
\hline $204.8+/-1.3$ & $204.8+/-1.3$ \\
\hline $199.6+/-1.3$ & $199.9+/-1.3$ \\
\hline $205.7+/-1.3$ & $206.2+/-1.3$ \\
\hline $205.8+/-1.3$ & $206.2+/-1.3$ \\
\hline $149.6+/-0.4$ & $150.2+/-1.3$ \\
\hline $141.7+/-0.4$ & $142.4+/-1.3$ \\
\hline $148.4+/-0.4$ & $148.5+/-1.3$ \\
\hline $99.4+/-0.2$ & $100.4+/-1.4$ \\
\hline $99.4+/-0.2$ & $100.4+/-1.4$ \\
\hline $90.8+/-0.2$ & $92.2+/-1.4$ \\
\hline $101.3+/-0.2$ & $102.4+/-1.4$ \\
\hline $69.6+/-0.1$ & $71.8+/-1.5$ \\
\hline $66.8+/-0.1$ & $68.8+/-1.5$ \\
\hline $66.9+/-0.1$ & $68.8+/-1.5$ \\
\hline $69.4+/-0.1$ & $71.8+/-1.5$ \\
\hline $54.6+/-0.1$ & $57.7+/-1.5$ \\
\hline $51.0+/-0.1$ & $51.5+/-1.6$ \\
\hline $48.4+/-0.1$ & $47.7+/-1.6$ \\
\hline
\end{tabular}

Tab. 3 summarizes the proton flux at the DUT as a function of energy for the two test stations. A beam spot size of $2.5 \mathrm{~cm}$ diameter is assumed. The strong energy dependence for RERS2 is a consequence of the momentum selection downstream of the degrader. The weak energy dependence of the flux in RERS1 is due to the lateral spreading of the beam downstream of the degrader. The table assumes that 50nA are extracted from the cyclotron.

Obviously, there is a trade-off of narrow beam energy spread vs intensity. The method of choice to degrade the beam energy depends on the application and whether high flux or small energy spread is the prime concern.

\section{BEAM PROFILES}

Beam diameters between 2 and $30 \mathrm{~cm}$ are achieved by the appropriate spreader in conjunction with a drift between the exit window and the DUT. For spot sizes up to $7 \mathrm{~cm}$ diameter a $\mathrm{Cu}$ foil (Fig.2, (2)) between 0.023 and $0.24 \mathrm{~mm}$ thickness is used while a $3 \mathrm{~mm}$ thick Ta foil (Fig.2, (3)), is used for beam diameters up to $30 \mathrm{~cm}$. At $205 \mathrm{MeV}$ the multiple scattering angle of Ta is twice as large than that for $\mathrm{Cu}$, thus increasing the geometric spreading significantly. The presence of the spreader also degrades the beam energy. If the Ta spreader is used, the beam energy is $190 \mathrm{MeV}$.

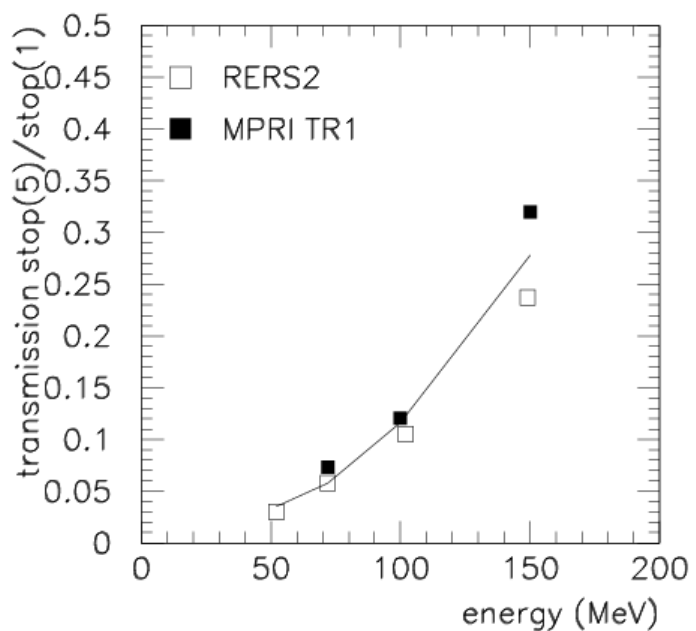

Figure 4. Transmission from stop (1) to the Faraday Cup (5). As a function of degraded beam energy. The solid black squares represent the transmission in the first medical beamline (TR1).

TABLE III. FLUXES FOR A 2.5 CM DIAMETER BEAMSPOT AS A FUNCTION OF ENERGY FOR BOTH BEAMLINES AND FOR A DAYTIME BEAM CURRENT OF 50 NA OUT OF THE CYCLOTRON. HIGHER FLUXES ARE AVAILABLE AT NIGHT WHEN HIGHER BEAM CURRENTS ARE ALLOWED.

\begin{tabular}{|c|c|c|}
\hline Energy (MeV) & $\begin{array}{c}\text { Flux }\left(\mathrm{p} / \mathrm{s} / \mathrm{cm}^{2}\right) \\
\text { RERS1 }\end{array}$ & $\begin{array}{c}\text { Flux }\left(\mathrm{p} / \mathrm{s} / \mathrm{cm}^{2}\right) \\
\text { RERS2 }\end{array}$ \\
\hline 200 & $1.5 \mathrm{e}^{10}$ & $1.5 \mathrm{e}^{10}$ \\
\hline 149 & $1.4 \mathrm{e}^{10}$ & $3.0 \mathrm{e}^{9}$ \\
\hline 102 & $1.3 \mathrm{e}^{10}$ & $7.4 \mathrm{e}^{8}$ \\
\hline 72 & $1.1 \mathrm{e}^{10}$ & $3.0 \mathrm{e}^{8}$ \\
\hline 52 & $1.1 \mathrm{e}^{10}$ & $1.6 \mathrm{e}^{8}$ \\
\hline
\end{tabular}

After passing through the spreader, the beam is confined by collimators. The beam diameter depends on the drift distance between the end of the beamline and the DUT. The DUT is either placed about $30 \mathrm{~cm}$ downstream of the exit window or it is placed near the beam dump, $336 \mathrm{~cm}$ from the exit window. The long drift between exit window and DUT allows the beam spot to expand to $30 \mathrm{~cm}$ diameter. Proton fluxes of the large area beam are as high as $10^{9}$ protons $/ \mathrm{s} / \mathrm{cm}^{2}$. The large area beam is useful for radiation effects tests of entire electronic systems. It has also been suggested that the large area beam may be used to simulate low-dose, long-term irradiation of self-contained biological systems such as rodent habitats [8].

Beam profiles at the location of the DUT are measured by irradiating GAFCHROMIC ${ }^{\circledR}$ film type HD 810 [9] to 30 krad. The resulting change in optical density of the film is measured using a Tobias model TBX densitometer with an interference filter whose band pass is $10 \%$ centered at 600 

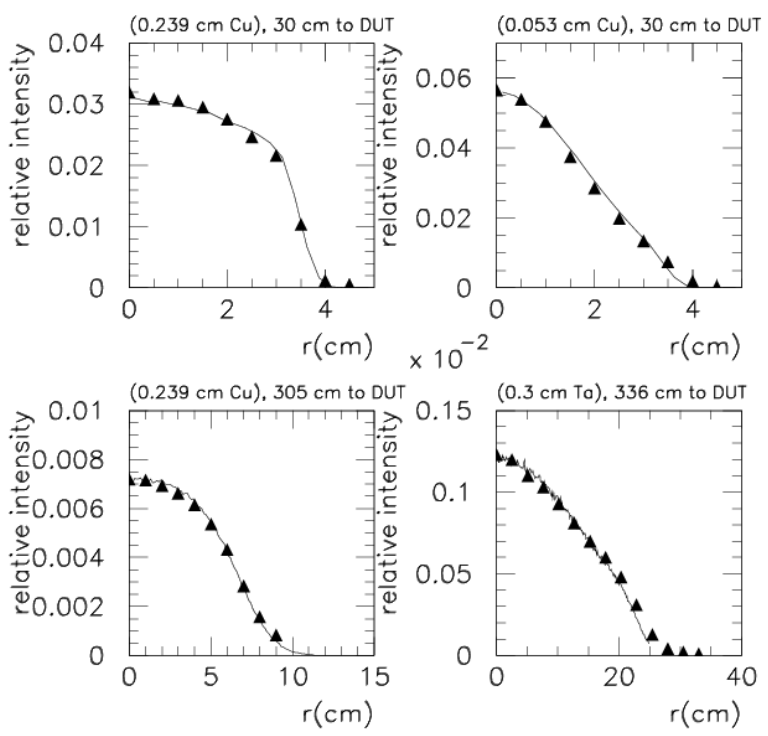

Figure 5. Beam profiles measured in RERS1 at $200 \mathrm{MeV}$. The triangles are data, the lines are simulations, details are given in the text.

nanometers. Representative samples of these beam profiles shown in Fig. 5. The ratio plotted in this figure is the average dose (film) in a square centimeter area at a radius, $r$, from the beam center divided by the total dose (film) integrated over the area of the beam. Beam intensity profiles show an intensity fall off of less than $40 \%$ from the center to the edge of the beam spot. The dosimetry software uses the central intensity of the dose profile and the current from the SEM to determine the fluence of protons on the DUT.

Fig. 5 shows measured profiles (triangles) for RERS1 at $205 \mathrm{MeV}$ using two different $\mathrm{Cu}$ spreader thicknesses as well as the Ta spreader. The profiles were taken $30 \mathrm{~cm}$ and 336 $\mathrm{cm}$ downstream of the exit window. Also shown are predicted profiles (lines) generated by a Monte Carlo simulation. Input to the simulation are beam energy, the thicknesses and materials of spreader, SEM and exit window along with collimater sizes and positions of all the components along the beam axis. The effects of multiple scattering and energy loss due to materials exposed to the beam are calculated. The simulation agrees with the measured profiles quite well. The simulation is not limited to RERS1 but reproduces profiles measured in RERS2 and at energies other than $205 \mathrm{MeV}$ equally well.

\section{DOSIMETRY}

The method of dosimetry has been described elsewhere [10]. Here we concentrate on new aspects of the dosimetry.

Since the SEM is located downstream of the Be degrader in RERS2, the energy dependence of the calibration factor relative to the Faraday Cup was investigated. The secondary electron current is proportional to the energy deposited in the SEM (dE/dx) [11]. The Faraday Cup (FC) measures the direct beam current and does not depend on energy. Therefore, the ratio of FC to SEM current should be

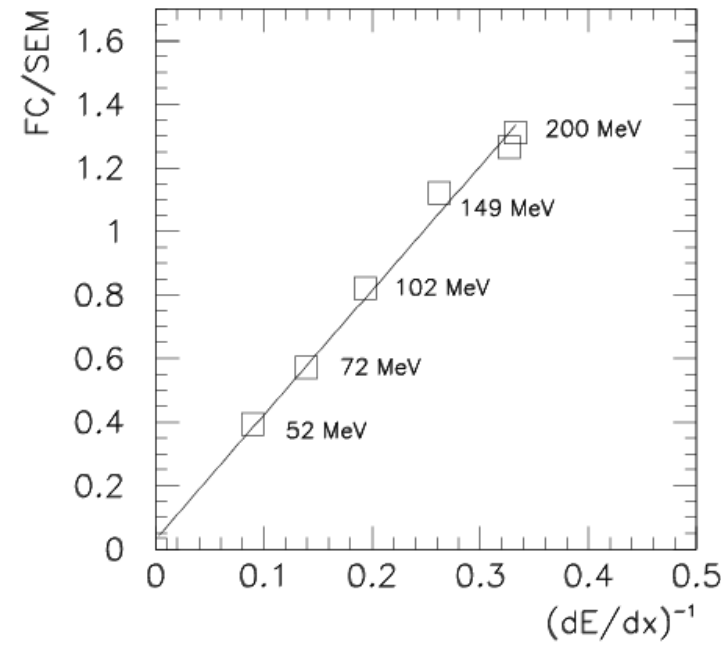

Figure 6: Energy dependence of the Faraday Cup (5) to SEM (4) ratio for RERS2.

inversely proportional to the energy loss in copper. The open squares in Fig. 6. are measurements of the FC/SEM ratio at $205 \mathrm{MeV}$ and at the four degraded beam energies and plotted as a function of $(\mathrm{dE} / \mathrm{dx})^{-1}$. The line is a linear fit which describes the energy dependence of the FC/SEM ratio quite well.

It is possible to compare the dose obtained from a film measurement with the dose obtained from the dosimetry software while the film is irradiated.

Samples of GAFCHROMIC film, type 810, were irradiated at the position of the DUT. They were irradiated at varying fluences to accumulate doses $\left(\mathrm{H}_{2} \mathrm{O}\right)$ from about 5 $\mathrm{krad}$ to about $50 \mathrm{krad}$. Films were irradiated using the standard beam spot in both beamlines, for varying energies in RERS2 and also for the new, large area beam spot in RERS1. Since the optical density of the GAFCHROMIC film increases with time reaching saturation less than 24 hours after irradiation, the films were read $24 \mathrm{~h}$ after irradiation.

In order to obtain an entirely independent verification of both the RERS dosimetry and the film calibration, films were also irradiated in the medical beamline (TR1) using the dosimetry software normally used for patient treatment. The film calibration used was the one provided by the manufacturer of the film.

Results of the measurements are summarized in Fig. 7. It should be noted that the RERS data points shown were taken during runs where beam was delivered to users, i.e. the graph represents average conditions. The error of each measurement is about $5 \%$.

\section{SUMMARY}

The radiation effects research program at IUCF is an active program which provides convenient, year-round access to high energy protons along with dosimetry over a 


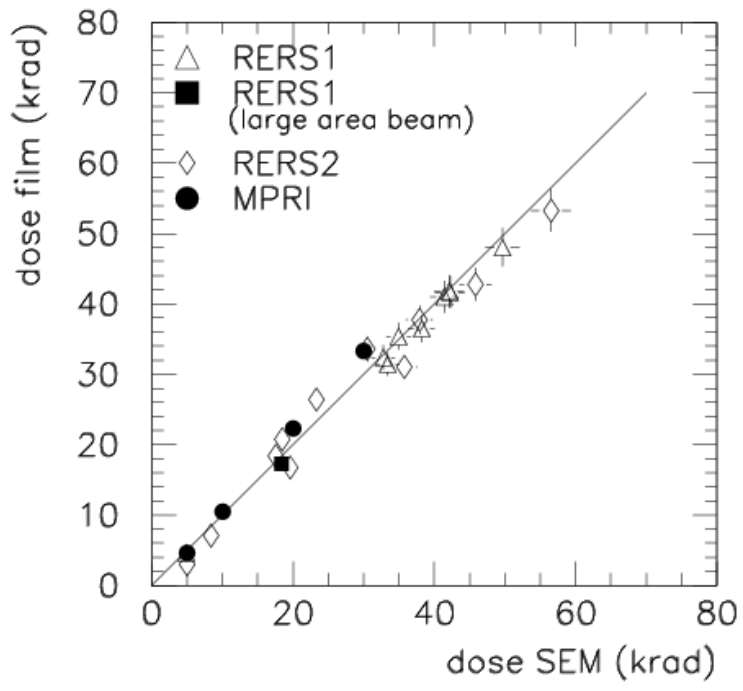

Figure 7: Dose measured with GAFCHROMIC film vs dose measured with the SEM. The line marks equal dose from either method.

wide range of proton fluxes. Two new beamlines, RERS1 and RERS2, are operational. Measured beam properties agree with model calculations. The dosimetry was verified independently and found to be consistent over the available range of energies.

\section{Future FACILITIES: THE LENS/NREP PROJECT}

As early as 2005, low energy neutron beams will become available at IUCF for radiation effects and materials testing. The facility envisioned in this project is a set of two neutron sources fed by a common proton accelerator, each optimized for separate functions [12].

Neutrons are produced by the proton beam impinging on a beryllium target. The Low Energy Neutron Source (LENS) will be optimized for production of "cold" neutrons for the development of neutron scattering instrumentation and materials research. The Neutron Radiation Effects Program (NREP) source will be optimized for production of neutrons with energies to simulate neutrons produced by nuclear events and by cosmic showers. Phase I (2005) will make use of an existing $7 \mathrm{MeV}$ proton accelerator. During phase II the existing accelerator will be replaced with a new, klystronbased $13 \mathrm{MeV}$ accelerator. With a $13 \mathrm{MeV}$ proton beam of $100 \mathrm{~mA}$ peak current and an accelerator duty factor of 5\% the expected neutron yield from the ${ }^{9} \mathrm{Be}$ target is $3 \times 10^{14} \mathrm{~s}^{-1}$.

\section{REFERENCES}

[1] E.L. Petersen, "The SEU Figure of Merit and Proton Upset Rate Calculations," IEEE Trans. Nuc. Sci. vol. 45, pp. 2550-2562, 1998.

[2] P.M. O'Neill, G.D. Badhwar, and W.X .Culpepper, "Internuclear Cascade-Evaporation Model for LET Spectra of $200 \mathrm{MeV}$ Protons Used for Parts Testing," IEEE Trans. Nuc. Sci., vol. 45, pp. 24672474,1998

[3] http://www.iucf.indiana.edu/RERP

[4] D.L. Friesel, T. Ellison and T. Sloan, "Beam Splitting Development," 1986 IUCF Scientific and Technical Report, p. 143
[5] Foster, C. C., et al., "Radiation Effects Test Facility at the Indiana University Cyclotron Facility," in Application of Accelerators in Research and Industry 1996, edited by J. L. Duggan and I. L. Morgan, AIP Conference Proceedings 392, New York, 1997, pp. 1131-1134.

[6] D.E. Groom et al., The European Physics Journal C15 (2000) 1

[7] TRIM, J.F. Ziegler and J. P. Biersack, http://www.srim.org

[8] S. Klein, private communication

[9] "GAFCHROMICTM " is a trademark of GAF Chem. Corp.

[10] Jones, A. Z., et al., IEEE Trans. Nuc. Sci., vol. 46, pp. 1762-1765, 1999.

[11] E.J. Sternglass, Physical Review 108 (1957) 1

[12] http://www.iucf.indiana.edu/RERP/rerp/neutron.shtml 